\title{
Erratum
}

\section{Erratum to: Diagnostic Value of Mini-laparoscopy in Patients with Abdominal Neoplasm}

Jian Wang, Yan-jun Ni, Shi-yao Chen ${ }^{*}$

Department of Gastroenterology and Hepatology, Zhongshan Hospital, Fudan University, Shanghai 200032, China

DOI: 10.1007/s11670-011-0323-9

(C) Chinese Anti-Cancer Association and Springer-Verlag Berlin Heidelberg 2011

Erratum to: Chin J Cancer Res 23(3):214-217, 2011

DOI: $10.1007 / \mathrm{s} 11670-011-0214-0$

The spelling of the author's department name in the original version of this article was incorrect. "Hepotology" should be corrected to "Hepatology". The correct information is given below:

Department of Gastroenterology and Hepatology

The online version of the original article can be found at

http:/ /dx.doi.org/10.1007/s11670-011-0214-0 